directly from sunlight. Highlights of the results included a top-tier catalyst for water oxidation using ultrahighthroughput methods and the stabilization (for a record long time and at record efficiencies) of a class of semiconductors for efficient water oxidation using earthabundant catalysts. Lewis also reported the development of membranes suited for the safe and efficient operation of a solar fuel generator and the construction of prototype systems that demonstrate the technology.

We look forward to the MRS Fall Meeting, which this year will be held in Boston, Massachusetts (USA) from November 30 to December 5, to learn about the latest developments in solar cells and other photonics topics related to materials science.

David Pile is at Nature Photonics, 225 Bush Street, Suite 1850, San Francisco, California 94104, USA. e-mail:d.pile@us.nature.com

\title{
MICRO-OPTICS
}

\section{Beetle-like microlens array}

Arrays of microlenses are widely used in various applications, including optical lab-on-a-chip devices, storage systems and photovoltaic devices. Presently employed methods for fabricating such arrays are restricted to flat substrates and are time consuming as they involve multiple steps. Now, Chloé Bayon, Gonzague Agez and Michel Mitov at Centre National de la Recherche Scientifique (CNRS) in Toulouse, France, have developed a single-step fabrication process based on self-assembly that can be used to produce cholesteric liquid-crystal microlens arrays on flat and curved substrates and sheets of rollable plastic (Lab Chip 14, 2063-2071; 2014). Importantly, the focusing and beamshaping properties of the microlenses are wavelength tunable.

Unlike conventional lenses in which the shape of lens provides the focusing effect, these latest microlenses rely on the intrinsic helical organization of the liquid crystal to perform beam shaping. The microlenses consist of polygons of cholesteric liquid crystal, where each polygon behaves as a one-dimensional photonic crystal (a Bragg grating) with a period determined by the pitch of the helical liquid crystal. The microlens arrays resemble some naturally occurring structures, such as the carapace of the beetle Chrysina gloriosa.

The arrays are fabricated by annealing and then rapidly quenching a blend

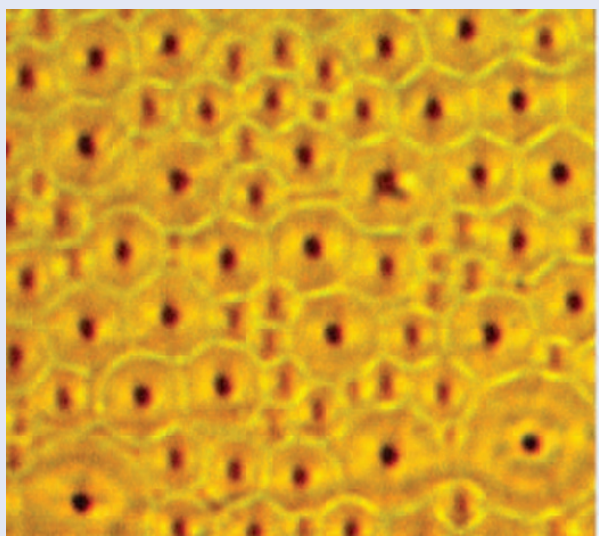

of cholesteric liquid-crystal oligomers. A polygonal array (see left panel of image) forms during the annealing step; this structure is then frozen by the quenching step.

The researchers experimentally analysed the optical properties of the microlenses using a technique that combines confocal microscopy and spectrophotometry. They identified three spectral regions of operation associated with the liquid crystal's structure: the Bragg band, the band edge and the red band. In the Bragg band and the band edge, the focal plane lies beyond the array, so that the array functions as a focusing lens. In contrast, in the red band, the focal plane lies in the plane of the array and produces a doughnut-shaped light intensity. The Bragg

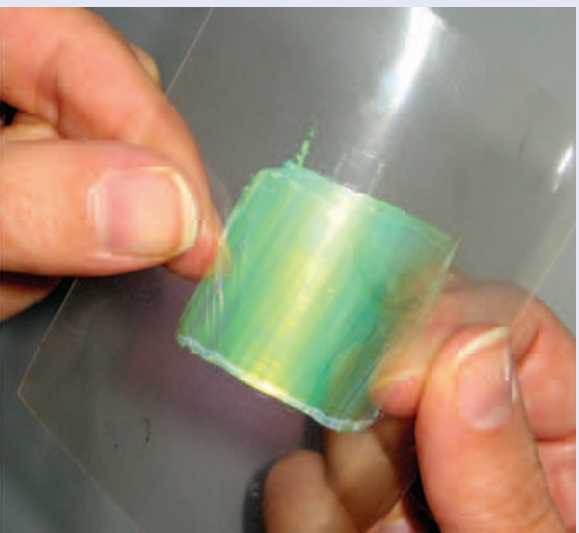

band and the band edge of the arrays became increasingly blue-shifted with longer annealing times. The scientists also conducted a numerical simulation of the array.

The team leader, Michel Mitov, told Nature Photonics that "the array is a versatile microdevice that could be integrated as a part of a lab-on-a-chip optical system" and that "it offers the combined benefits of multiple light manipulation capabilities, seamless integration and mechanical stability." In the future, the team intends to investigate whether doping the liquid crystals with a dye could be used to produce a microlaser array.

\section{SIMON PLEASANTS}

